\title{
Responses of inflammatory cytokines following moderate intensity walking exercise in overweight or obese individuals
}

\author{
Yunsuk Koh'1, Kyung-Shin Park ${ }^{2, *}$ \\ ${ }^{1}$ Health Human Peprformance Recreation, Baylor University, Waco, TX, USA \\ ${ }^{2}$ Kinesiology, Texas A\&M International University, Laredo, TX, USA
}

This study investigated the effects of a 4-week moderate intensity walking exercise on the levels of $\mathrm{C}$-reactive protein (CRP), tumor necrosis factor-alpha (TNF- $\alpha$ ), and adiponectin in overweight or obese individuals. Twenty-seven (13 men and 14 women) physically inactive, overweight or obese (body mass index $>25.0 \mathrm{~kg} / \mathrm{m}^{2}$ ) individuals participated in the study. Each participant was randomly assigned to either exercise $(E X ; n=15)$ or control (CON; $n=12$ ) group. The EX group performed moderate intensity walking exercise on a treadmill for $60 \mathrm{~min}$ at $70 \%$ of maximal heart rate for 4 weeks ( 3 days/wk). Overnight fasting blood samples were collected before and after the study period (Pre and Post) to analyze the levels of pro-/anti-inflammatory cytokines such as CRP, TNF- $\alpha$, and adiponectin. CRP and adiponectin as well as body weight and body composition were not significantly altered following the 4-week exercise intervention. However, there was a significant group $\times$ time interac- tion for TNF- $\alpha$. The post hoc test revealed that the level of TNF- $\alpha$ significantly decreased only in EX (EX: mean \pm standard deviation, Pre $55.18 \pm 6.57 \mathrm{pg} / \mathrm{mL}$, Post $50.31 \pm 6.22, P=0.018$ vs. CON: Pre $55.5 \pm 5.88$, Post $58.19 \pm 6.48, P=0.25)$. EX Post was also significantly lower as compared to CON Post $(P=0.0074)$. Although there was no change in body weight or fat mass, the 4-week aerobic exercise training was long enough to decrease the level of TNF- $\alpha$, indicating physical activity may improve level of inflammation independently from the change in fat mass. The current study also confirmed previous research suggesting that longer period of exercise training accompanied by weight loss may be required to induce significant changes in CRP and adiponectin.

Keywords: Aerobic exercise, Body composition, C-reactive protein, Tumor necrosis factor-alpha, Adiponectin

\section{INTRODUCTION}

Recently, fat cells, especially visceral fat and central body fat (BF), are known not only as a simple energy depository tissue, but also as an active endocrine organ releasing a variety of active molecules known as adipokines (Beavers et al., 2013; Bruun et al., 2006; de Ferranti and Mozaffarian, 2008). Excess visceral fat cell releases more inflammatory cytokines such as $\mathrm{C}$-reactive protein (CRP) and tumor necrosis factor-alpha (TNF- $\alpha$ ) and elevates the level of oxidative stress, leading to insulin resistance in skeletal muscles which may induce diabetes, (Bastard et al., 2006). Furthermore, central obesity is associated with a decreased production of adiponectin, antidiabetic, antiatherosclerotic and anti-inflam-

matory molecule, which causes chronic disease (Matsuzawa et al., 2004). Thus, the inflammatory pathway is a potential therapeutic target for lifestyle interventions designed for the obese population to reduce disease and disability (You et al., 2004).

Chronic aerobic exercise has been considered as one of the effective and preventive countermeasure to chronic diseases. Results of previous studies indicated that regular aerobic exercise improves many components of the cardiovascular risk factor as well as insulin sensitivity (Beavers et al., 2013; Blair and Brodney, 1999; Kelly et al., 2007; Nassis et al., 2005) by enhancing a variety of metabolic abnormalities and decreasing cardiovascular risk factors. Therefore, it is plausible that regular aerobic exercise may improve the inflammatory profile independently from weight loss. Previ-

*Corresponding author: Kyung-Shin Park (D) https://orcid.org/0000-0002-0761-4502 Kinesiology, Texas A\&M International University, 5201 University Blvd., Laredo, TX, USA

Tel: +1-956-473-9585, Fax: +1-956-326-2434, E-mail: kpark@tamiu.edu Received: July 3, 2017 / Accepted: August 12, 2017 
ous studies found reductions in pro-inflammatory cytokines (Abramson and Vaccarino, 2002; Adamopoulos et al., 2001; Church et al., 2002; Conraads et al., 2002; Mattusch et al., 2000; Tsukui et al., 2000), indicating that long-term exercise training may reduce chronic low-level inflammation in overweight and obese population. However, the more recent studies failed to confirm the effects of exercise on inflammatory cytokines (Beavers et al., 2013; Kelly et al., 2007; Marcell et al., 2005), possibly due to not isolating the effects of exercise training from weight loss. In addition, the mechanism which links exercise training to these inflammatory markers is not fully understood yet.

With given information of the relation between obesity and cardiovascular risk factors, insulin resistance, and inflammation, it is important to understand whether systemic inflammation can be in part explained by the physiologic benefits of exercise and whether exercise can be used as a potential therapeutic intervention for overweight and obese individuals. Therefore, we designed a short-term exercise training intervention to investigate effects of exercise training on inflammatory biomarkers in the absence of weight loss and fat loss.

\section{MATERIALS AND METHODS}

\section{Participants}

Twenty-seven overweight or obese (body mass index [BMI] $\left.>25.0 \mathrm{~kg} / \mathrm{m}^{2}\right)$ men $(\mathrm{n}=13)$ and women $(\mathrm{n}=14)$, ages between 18 and 65 years, participated in the study. All participants were physically inactive; did not have any known chronic disease; did not take medication that would alter metabolic, cardiovascular, or immune function; and had no musculoskeletal limitations. This study was approved by the Institutional Review Board and the participants provided written informed consent and the medical history forms prior to performing any study protocols. The participants maintained a normal dietary regimen and refrained from any forms of aerobic or anaerobic physical activity other than the exercise treatment throughout the study period.

\section{Study design}

\section{Exercise intervention}

During the initial visit, the participants were randomly assigned to either exercise (EX; $n=15)$ or control $(\mathrm{CON} ; \mathrm{n}=12)$ group and anthropometric and physiological variables at baseline were then measured (Table 1). Additionally, the participants that were assigned to the EX group conducted a treadmill exercise test to determine the appropriate exercise intensity (70\% of age-predicted maximal heart rate $[H R \max ], 0.7 \times[220$ - age]). During the initial treadmill exercise testing, the participants were allowed to choose their own desirable and comfortable walking speed, which was maintained throughout the testing. However, the grade (\%) was adjusted every 2 min until the participants reached their target exercise intensity (70\% of HRmax). Once the participants reached their target exercise intensity, the speed and grade were recorded, and the testing was terminated. As for the exercise intervention, the participants in the EX group performed walking exercise on the treadmill at predetermined exercise intensity $(70 \%$ HRmax) for 60 min, 3 days a week for 4 weeks. During each exercise session, the participants'heart rate was monitored using the Polar heart rate monitor (Polar Electro Inc., Lake Success, NY, USA) and recorded every $10 \mathrm{~min}$ to ensure their target exercise intensity. All exercise sessions were supervised by research assistants. The participants in the CON group did not perform any physical activity throughout the study period.

\section{Measurement of body composition}

Body composition including muscle mass, fat mass, and \% BF were measured using a bioelectrical impedance analyzer (BIA101A, RJL Systems, Clinton Township, MI, USA). The participants refrained from physical activity, sauna, or alcohol consumption within $12 \mathrm{hr}$ prior to the measurement of body composition. During the measurement, the participants lay supine on the floor

Table 1. Changes in anthropometric characteristics and body composition

\begin{tabular}{|c|c|c|c|c|}
\hline \multirow{2}{*}{ Variable } & \multicolumn{2}{|c|}{$E X(n=15)$} & \multicolumn{2}{|c|}{$\operatorname{CON}(n=12)$} \\
\hline & Pre & Post & Pre & Post \\
\hline Height (cm) & $165.7 \pm 10.3$ & - & $171.1 \pm 7.7$ & - \\
\hline Weight (kg) & $90.33 \pm 23.05$ & $90.21 \pm 22.97$ & $94.82 \pm 12.53$ & $94.81 \pm 12.67$ \\
\hline $\mathrm{BMI}\left(\mathrm{kg} / \mathrm{m}^{2}\right)$ & $33.24 \pm 5.67$ & $32.91 \pm 5.73$ & $32.54 \pm 5.28$ & $32.58 \pm 5.23$ \\
\hline Body fat (\%) & $35.42 \pm 8.91$ & $34.62 \pm 9.12$ & $34.50 \pm 9.99$ & $35.61 \pm 9.82$ \\
\hline
\end{tabular}

Values are presented as mean \pm standard deviation.

EX, exercise group; CON, control group; BMI, body mass index. 
with arms away from the body with no shoes, socks, or any jewelry, and electrodes were placed on a hand and foot as instructed by the manufacture's procedure. The participants remained lying on the floor until the completion of the test and then resistance and reactance were recorded, which were later used to estimate muscle mass, fat mass, and \% BF using Body Composition software (BC 2.1, RJL Systems, Clinton Township, MI, USA).

\section{Blood sample collection}

Five milliliters of overnight fasting (10-12 hr) blood samples were collected at pre- and poststudy. After $10 \mathrm{~min}$ of resting in a chair, venous blood from the antecubital vein was collected into a serum tube, remained at room temperature for $20 \mathrm{~min}$ to be clotted, and then was centrifuged $(1,000 \mathrm{~g})$ at $4^{\circ} \mathrm{C}$ for $20 \mathrm{~min}$ to separate serum. Serum samples were allocated into $1.5 \mathrm{~mL}$ tubes and immediately frozen at $-80^{\circ} \mathrm{C}$ for the further analyses.

\section{Blood analyses}

Serum samples in duplicate were assayed via the enzyme-linked immunosorbent assay (Boster Biological Technology Co., Ltd., Pleasanton, CA, USA) to measure blood adiponectin, TNF- $\alpha$, and CRP concentrations. Optical density was measured by the microplate reader (EL 808, BioTek Co, Winooski, VT, USA). The mean intra-assay coefficient of variabilities (CVs) were $6.9 \%, 4.3 \%$, and $5.3 \%$ and inter-assay CVs were $8.2 \%, 6.7 \%$, and $7.8 \%$ for adiponectin, TNF- $\alpha$, and CRP, respectively.

\section{Data analysis}

The sample size was calculated by $G *$ Power 3.1.0 software, given an alpha level at 0.05 , an effect size of 0.40 , and power at 0.80 . The appropriate sample size was estimated to be 21 participants for the current study. All statistical analyses were conducted using Sigmaplot 13 (Systat Software, Inc., San Jose, CA, USA). Two-way analysis of variance with repeated measures was used to analyze changes in BMI, body composition, and inflammatory cytokines. Post hoc tests were performed using Tukey post hoc test and statistical significance was set at $P<0.05$.

\section{RESULTS}

Physical characteristics and body composition at baseline (Pre) and after the 4 weeks of moderate intensity walking exercise (Post) are shown up in Table 1. There were no significant differences found between EX and CON groups at Pre. Body weight, BMI, and $\% \mathrm{BF}$ were unaltered following the 4 weeks of moderate intensity walking exercise.

Changes in inflammatory cytokines are found in Table 2. Although there was a noticeable decrease in CRP following the 4 weeks of moderate intensity walking exercise, it was not statistically significant (EX: mean \pm standard deviation [SD], Pre $6.38 \pm 2.33 \mu \mathrm{g} / \mathrm{mL}$, Post $5.11 \pm 1.78 \mu \mathrm{g} / \mathrm{mL}, P=0.062$ vs. CON: Pre $6.44 \pm 1.77 \mu \mathrm{g} / \mathrm{mL}$, Post $6.54 \pm 1.88 \mu \mathrm{g} / \mathrm{mL}, P=0.76$ ). There was no significant group $\times$ time interaction found in the level of adiponectin (EX: mean \pm SD, Pre $7.1 \pm 1.3 \mu \mathrm{g} / \mathrm{mL}$, Post $7.2 \pm 1.3$ $\mu \mathrm{g} / \mathrm{mL}, P=0.87$ vs. CON: Pre $9.8 \pm 1.5 \mu \mathrm{g} / \mathrm{mL}$, Post $9.9 \pm 1.5 \mu \mathrm{g} /$ $\mathrm{mL}, P=0.83)$ although $\mathrm{CON}$ showered higher value as compared to EX (group effect, $P=0.040$ ). However, there was a significant group $\times$ time interaction (Fig. 1) for TNF- $\alpha$ and the post hoc test

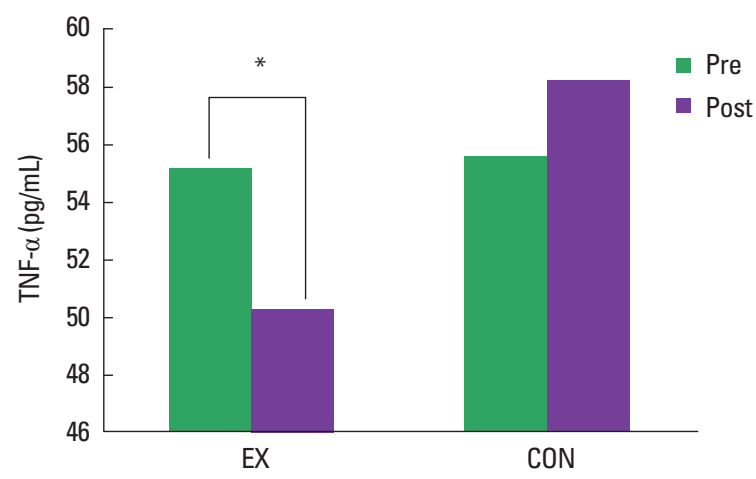

Fig. 1. A group by time interaction showing changes in tumor necrosis factor-alpha $(T N F-\alpha)$ Values are presented as mean \pm standard deviation. EX, exercise group; $\mathrm{CON}$, control group. ${ }^{*} P=0.018$.

Table 2. Changes in inflammatory cytokines

\begin{tabular}{|c|c|c|c|c|}
\hline \multirow{2}{*}{ Variable } & \multicolumn{2}{|c|}{$\operatorname{EX}(n=15)$} & \multicolumn{2}{|c|}{$\operatorname{CON}(n=12)$} \\
\hline & Pre & Post & Pre & Post \\
\hline C-reactive protein $(\mu \mathrm{g} / \mathrm{mL})$ & $6.38 \pm 2.33$ & $5.11 \pm 1.78$ & $6.44 \pm 1.77$ & $6.54 \pm 1.88$ \\
\hline Adiponectin ( $\mu \mathrm{g} / \mathrm{mL})$ & $7.1 \pm 1.3$ & $7.2 \pm 1.3$ & $9.8 \pm 1.5$ & $9.9 \pm 1.5$ \\
\hline TNF- $\alpha(p g / m L)$ & $55.18 \pm 6.57$ & $50.31 \pm 6.22^{*}$ & $55.5 \pm 5.88$ & $58.19 \pm 6.48$ \\
\hline
\end{tabular}

Values are presented as mean \pm standard deviation.

EX, exercise group; CON, control group; TNF- $\alpha$, tumor necrosis factor-alpha.

${ }^{*}$ Significantly different from Pre $(P=0.018)$, significantly different from CON Post $(P=0.0074)$. 
revealed that TNF- $\alpha$ significantly decreased only in EX (EX: mean \pm SD, Pre $55.18 \pm 6.57 \mathrm{pg} / \mathrm{mL}$, Post $50.31 \pm 6.22, P=0.018$ vs. CON: Pre 55.5 \pm 5.88 , Post $58.19 \pm 6.48, P=0.25)$. EX Post is also significantly lower as compared to CON Post $(P=0.0074)$.

\section{DISCUSSION}

The current study investigated the effects of a 4-week moderate intensity walking exercise on levels of pro-/anti-inflammatory cytokines in overweight or obese individuals in the absence of weight loss. The 4-week exercise training resulted in no changes in fat mass, CRP, or adiponectin, whereas TNF- $\alpha$ was significantly reduced.

Physical inactivity and obesity have been considered as independent risk factors for cardiovascular diseases and metabolic syndrome, which may contribute to an increase in circulating inflammatory markers (Abramson and Vaccarino, 2002; Adamopoulos et al., 2001; Beavers et al., 2013; Blair and Brodney, 1999; Bruun et al., 2006; de Ferranti and Mozaffarian, 2008). However, the effects of regular exercise on inflammation are inconclusive due to lack of evidence.

You et al. (2004) found that the diet plus exercise intervention decreased CRP and TNF- $\alpha$, while the diet only group failed to show the same result although the similar amount of reduction in fat mass was observed. The result corresponded to other previous research (Abramson and Vaccarino, 2002; Adamopoulos et al., 2001; Mattusch et al., 2000; Straczkowski et al., 2001; Tsukui et al., 2000). Other studies found that the levels of pro-inflammatory cytokines are in an inverse relationship with aerobic fitness (Church et al., 2002; LaMonte et al., 2002); however, the same effect has not uniformly been seen in all intervention studies.

Beavers et al. (2015) reported that the reductions of inflammatory cytokines are not achieved by exercise intervention but are directly associated with loss of fat mass, suggesting that significant reductions of inflammatory cytokines can be obtained with more than $5 \%$ loss of fat mass. It was correspondent to Nicklas et al. (2004) and Marcell et al. (2005), which added that changes in adiponectin is also associated with weight loss instead of improvement in fitness and concluded that regular exercise does not change the levels of inflammatory cytokines. In addition, most of the studies that reported significant changes in inflammatory cytokines by exercise training found weight loss; therefore, it is not clear whether changes in inflammatory cytokines following exercise training is caused by physiological benefits of exercise training or by weight loss accompanied to exercise.
In the present study, CRP and adiponectin did not change following the 4 weeks of moderate intensity exercise training. It is probable that changes in CRP and adiponectin may require a certain duration of exercise training or at least moderate amount of weight loss as shown in other studies (Bouassida et al., 2010; Hulver et al., 2002; Marcell et al., 2005; Ryan et al., 2003; Shephard, 2002).

In conclusion, the present study suggests that TNF- $\alpha$ can be lowered following the 4 weeks of moderate intensity exercise training in overweighed and obese individuals without a significant reduction in weight or fat mass, indicating physical activity independently from a change in fat mass may affect the level of inflammation. The present study also confirmed the previous studies suggesting that longer period of training may be required to induce a significant change in CRP or adiponectin. It is recommended that additional evidence be sought to elucidate the independent role of aerobic exercise training for chronic inflammation in overweight and obese individuals.

\section{CONFLICT OF INTEREST}

No potential conflict of interest relevant to this article was reported.

\section{ACKNOWLEDGMENTS}

This study was performed with an approval of Baylor University and supported by Texas A\&M International University Research Grant.

\section{REFERENCES}

Abramson JL, Vaccarino V. Relationship between physical activity and inflammation among apparently healthy middle-aged and older US adults. Arch Intern Med 2002;162:1286-1292.

Adamopoulos S, Parissis J, Kroupis C, Georgiadis M, Karatzas D, Karavolias G, Koniavitou K, Coats AJ, Kremastinos DT. Physical training reduces peripheral markers of inflammation in patients with chronic heart failure. Eur Heart J 2001;22:791-797.

Bastard JP, Maachi M, Lagathu C, Kim MJ, Caron M, Vidal H, Capeau J, Feve B. Recent advances in the relationship between obesity, inflammation, and insulin resistance. Eur Cytokine Netw 2006;17:4-12.

Beavers KM, Ambrosius WT, Nicklas BJ, Rejeski WJ. Independent and combined effects of physical activity and weight loss on inflammatory biomarkers in overweight and obese older adults. J Am Geriatr Soc 
2013;61:1089-1094.

Beavers KM, Beavers DP, Newman JJ, Anderson AM, Loeser RF Jr, Nicklas BJ, Lyles MF, Miller GD, Mihalko SL, Messier SP. Effects of total and regional fat loss on plasma CRP and IL-6 in overweight and obese, older adults with knee osteoarthritis. Osteoarthritis Cartilage 2015;23:249-256.

Blair SN, Brodney S. Effects of physical inactivity and obesity on morbidity and mortality: current evidence and research issues. Med Sci Sports Exerc 1999;31(11 Suppl):S646-662.

Bouassida A, Chamari K, Zaouali M, Feki Y, Zbidi A, Tabka Z. Review on leptin and adiponectin responses and adaptations to acute and chronic exercise. Br J Sports Med 2010;44:620-630.

Bruun JM, Helge JW, Richelsen B, Stallknecht B. Diet and exercise reduce low-grade inflammation and macrophage infiltration in adipose tissue but not in skeletal muscle in severely obese subjects. Am J Physiol Endocrinol Metab 2006;290:E961-967.

Church TS, Barlow CE, Earnest CP, Kampert JB, Priest EL, Blair SN. Associations between cardiorespiratory fitness and $\mathrm{C}$-reactive protein in men. Arterioscler Thromb Vasc Biol 2002;22:1869-1876.

Conraads VM, Beckers P, Bosmans J, De Clerck LS, Stevens WJ, Vrints CJ, Brutsaert DL. Combined endurance/resistance training reduces plasma TNF-alpha receptor levels in patients with chronic heart failure and coronary artery disease. Eur Heart J 2002;23:1854-1860.

de Ferranti S, Mozaffarian D. The perfect storm: obesity, adipocyte dysfunction, and metabolic consequences. Clin Chem 2008;54:945-955.

Hulver MW, Zheng D, Tanner CJ, Houmard JA, Kraus WE, Slentz CA, Sinha MK, Pories WJ, MacDonald KG, Dohm GL. Adiponectin is not altered with exercise training despite enhanced insulin action. Am J Physiol Endocrinol Metab 2002;283:E861-865.

Kelly AS, Steinberger J, Olson TP, Dengel DR. In the absence of weight loss, exercise training does not improve adipokines or oxidative stress in overweight children. Metabolism 2007;56:1005-1009.

LaMonte MJ, Durstine JL, Yanowitz FG, Lim T, DuBose KD, Davis P, Ainsworth BE. Cardiorespiratory fitness and C-reactive protein among a tri-ethnic sample of women. Circulation 2002;106:403-406.

Marcell TJ, McAuley KA, Traustadóttir T, Reaven PD. Exercise training is not associated with improved levels of C-reactive protein or adiponectin. Metabolism 2005;54:533-541.

Matsuzawa Y, Funahashi T, Kihara S, Shimomura I. Adiponectin and metabolic syndrome. Arterioscler Thromb Vasc Biol 2004;24:29-33.

Mattusch F, Dufaux B, Heine O, Mertens I, Rost R. Reduction of the plasma concentration of C-reactive protein following nine months of endurance training. Int J Sports Med 2000;21:21-24.

Nassis GP, Papantakou K, Skenderi K, Triandafillopoulou M, Kavouras SA, Yannakoulia M, Chrousos GP, Sidossis LS. Aerobic exercise training improves insulin sensitivity without changes in body weight, body fat, adiponectin, and inflammatory markers in overweight and obese girls. Metabolism 2005;54:1472-1479.

Nicklas BJ, Ambrosius W, Messier SP, Miller GD, Penninx BW, Loeser RF, Palla S, Bleecker E, Pahor M. Diet-induced weight loss, exercise, and chronic inflammation in older, obese adults: a randomized controlled clinical trial. Am J Clin Nutr 2004;79:544-551.

Ryan AS, Nicklas BJ, Berman DM, Elahi D. Adiponectin levels do not change with moderate dietary induced weight loss and exercise in obese postmenopausal women. Int J Obes Relat Metab Disord 2003; 27:1066-1071.

Shephard RJ. Cytokine responses to physical activity, with particular reference to IL-6: sources, actions, and clinical implications. Crit Rev Immunol 2002;22:165-182.

Straczkowski M, Kowalska I, Dzienis-Straczkowska S, Stepién A, Skibińska E, Szelachowska M, Kinalska I. Changes in tumor necrosis factor-alpha system and insulin sensitivity during an exercise training program in obese women with normal and impaired glucose tolerance. Eur J Endocrinol 2001;145:273-280.

Tsukui S, Kanda T, Nara M, Nishino M, Kondo T, Kobayashi I. Moderate-intensity regular exercise decreases serum tumor necrosis factor-alpha and $\mathrm{HbA1c}$ levels in healthy women. Int J Obes Relat Metab Disord 2000;24:1207-1211.

You T, Berman DM, Ryan AS, Nicklas BJ. Effects of hypocaloric diet and exercise training on inflammation and adipocyte lipolysis in obese postmenopausal women. J Clin Endocrinol Metab 2004;89:1739-1746. 